\title{
SPECIFICITY OF INTRINSIC CONNECTIONS IN PRIMATE PRIMARY VISUAL CORTEX ${ }^{1}$
}

\author{
MARGARE'T S. IIVINGSTONE ${ }^{2}$ AND DAVID H. HUBEI. \\ Department of Neurobiology, Harvard Medical School, Boston Massachusetts 02115
}

Received March 21, 1984; Revised May 14, 1984; Accepted May 30, 1984

\begin{abstract}
Several recent studies have suggested a patchy system of intrinsic lateral connections in area 17 of the macaque monkey. To see whether this pattern bore any relationship to the cytochrome oxidase blobs we made multiple tiny injections of horseradish peroxidase into layers 2 and 3 of area 17 , small enough so that some of the injections (or their cores) were entirely inside a single blob, or entirely outside. When the injection centers were entirely in blobs, the label in layers 2 and 3 was transported preferentially to nearby blobs, avoiding nonblob areas. When the injections were in nonblob areas, the label was found predominantly in surrounding nonblob areas, avoiding the blobs. Besides this lateral transport, label was also present in the layers below 2 and 3: the label in layers 4 and 6 was very restricted, occupying roughly the diameter of the injection core and presumably representing axons of cell bodies at the injection site; in layer 5 diffusely labeled patches observed the same blob/nonblob segregation seen above layer 4 .
\end{abstract}

The mammalian cortex is organized in such a way that cells sharing similar physiological properties tend to lie in vertical columns (Mountcastle, 1957; Hubel and Wiesel, 1962). It is therefore not surprising that most of the connections within the cortex run vertically (Lorente de Nó, 1933), presumably interconnecting cells with similar physiological properties. Gilbert and Wiesel (1979) found that the axons of single cells in area 17 of the cat extended farther horizontally than suspected from Golgi studies, for a distance much greater than the dimension of a single column. The branches of these axons were distributed in clusters. It is not known whether these horizontally running axons connect similar or dissimilar types of cells, but the fact that clusters from a single cell lay in vertical register in two different layers suggests a possible relationship to the physiologically defined columns (Gilbert and Wiesel, 1983).

In the cat, none of the classical anatomical methods reflect the columnar organization. In the monkey striate cortex, however, cytochrome oxidase staining reveals a quasiregular array of densely staining regions (blobs), which can be seen lying in register in the layers above and below layer 4 (Hendrickson et al., 1981; Horton and Hubel, 1981; Livingstone and Hubel, 1982). Cells within blobs are physiologically distinct from cells outside blobs in that they are not sensitive to the orientation of a visual stimulus but, rather, seem mostly concerned with its color properties (Livingstone and Hubel, 1984). Cells outside

\footnotetext{
${ }^{1}$ This work was supported by National Institutes of Health Grants RO1 EY00605 and EY01995, by the Klingenstein Fund, and by the Rowland Foundation. The histology was done by Debra Broihier and Janet Robbins, and the photography was by Marc Peloquin. We thank Jennifer Lund and Gary Blasdel for helpful discussions on making tiny horseradish peroxidase injections, and two reviewers for helpful comments on the manuscript.

${ }^{2}$ To whom correspondence should be addressed.
}

the blobs are selective for stimulus orientation and most (80 to $90 \%$ ) show no color specificity. Physiologically the two sets of cells thus have very little in common. Anatomically they are also very different, in that the blobs project selectively to a set of stripe-like regions in area 18 that stain darkly for cytochrome oxidase, whereas the nonblob regions of area 17 project to lighter staining regions of area 18 between the stripes (Livingstone and Hubel, 1984). The reciprocal 18-to-17 connections are likewise segregated. Furthermore, within area 18 the darkly staining regions connect preferentially to adjacent dark staining regions, skipping intervening light regions, and the lighter regions similarly are preferentially interconnected. We do not yet know what the differences are in the physiological properties of cells in dark and light staining regions of area 18.

It seemed natural to ask whether connections within area 17 similarly observed a segregation between the dark and light staining regions. Rockland and Lund (1983) found that even after relatively large extracellular horseradish peroxidase injections into area 17 they could see a patchiness in the transported label. The patches of label had roughly the same size and spacing as the cytochrome oxidase blobs. The label did not, however, correspond to the cytochrome oxidase blobs but rather formed a lattice that included the blobs in its walls. When we similarly made large injections, using wheat germ-conjugated horseradish peroxidase instead of ordinary peroxidase, we found that the transported label was mainly in blobs (Livingstone and Hubel, 1984). Despite this difference in the results, both studies suggest that the intrinsic connectivity in striate cortex bears some regular and strong relationship to the subdivision of the cortex into blob and nonblob regions.

\section{Materials and Methods}

We used four young adult female Macaca fascicularis monkeys. The monkeys were anesthetized with ketamine and 1.5 to $2 \%$ halothane, and many holes were drilled into the skull on each side, over area 17. 
Horseradish peroxidase (Boehringer Mannheim) dissolved in $0.1 \mathrm{M}$ Tris buffer, $\mathrm{pH} 8.6(5 \% \mathrm{w} / \mathrm{v})$, was then injected iontophoretically from glass micropipettes (10- $\mu \mathrm{m}$ tip diameter; $0.5 \mu \mathrm{A}$ for $2 \mathrm{~min}$; square wave pulses: $5 \mathrm{sec}$ on, $10 \mathrm{sec}$ off, electrode positive). In each hemisphere we made 15 to 25 injections, 0.1 to $0.3 \mathrm{~mm}$ below the pia. In one hemisphere of one monkey we used $5 \%$ wheat germ-conjugated horseradish peroxidase (Sigma Chemical Co.) instead of unconjugated horseradish peroxidase. After a 2-day survival time the monkeys were anesthetized with ketamine and halothane and perfused transcardially with fixative (2.25\% glutaraldehyde, $0.75 \%$ paraformaldehyde, $0.9 \% \mathrm{NaCl}, 0.1 \mathrm{M}$ sodium phosphate, $\mathrm{pH} 7.4$ ). The occipital lobes were flattened between two pieces of Teflon and frozen. Sections were cut tangentially at 50 $\mu \mathrm{m}$ thickness, and alternate sections were reacted for cytochrome oxidase (Wong-Riley, 1979) or for horseradish peroxidase using tetramethylbenzidine (Mesulam, 1982).

\section{Results}

Fortunately, there was no cross-reactivity between the horseradish peroxidase and the cytochrome oxidase stain. With the tetramethylbenzidine method of staining, the injection sites typically consisted of a tiny dense reddish core, 50 to $150 \mu \mathrm{m}$ in diameter, surrounded by a much larger (200 to $500 \mu \mathrm{m}$ ) yellowish halo. From present and previous studies (Livingstone and Hubel, 1984) our impression is that transport originates from the injection core rather than from the halo. Of the 112 injections made with unconjugated horseradish peroxidase, 97 were less than $150 \mu \mathrm{m}$ in diameter. Thirty-four of these were smaller than the rest and produced no transported label. The other 63 injection sites were surrounded by patches of transported label 100 to $200 \mu \mathrm{m}$ in diameter extending up to $1 \mathrm{~mm}$ from the center of the injection site. Of these 63 injections, 12 had their cores centered on blobs, 38 were clearly outside of blobs, 5 had their cores not centered in blobs but just at the edges, and the remaining 8 spanned both blob and nonblob regions. In the one hemisphere in which we used wheat germconjugated horseradish peroxidase, we saw no patches of label around the injection sites, even though these injections were similar in size to those in the other hemisphere.

Six horseradish peroxidase injections and adjacent cytochrome oxidase-stained sections are shown in Figures 1 and 2. These sections are all from layers $2-3$. The center of each injection is less than $150 \mu \mathrm{m}$ in diameter. Surrounding this is a dark halo up to 200 to $300 \mu \mathrm{m}$ in diameter, containing diffusely distributed grains and labeled cells. We do not know whether any of this label was actively transported from the core. Beyond the halo, one can see patches of diffuse transported label for a distance up to $1 \mathrm{~mm}$ from the injection site. None of the label in the patches was convincingly or obviously in cell bodies, and we assume that it represents orthograde transport. Thin lines of label, presumably axons, often fan out and bridge the distance between the injection site and the patches of label.

Figure $1(a$ to $c$ ) and the corresponding acetate overlays show injections that were outside blobs. The transported label for each of these injections also lay outside blobs. The label did not fill the entire interblob matrix but, rather, subregions of it.

Figure 1 ( $d$ to $f$ ) (in color and in the overlay) shows injections that were centered on blobs. The transported label looks very much like the label from the nonblob injections, but when the acetate overlay of the tetramethylbenzidine-reacted section is aligned with the cytochrome oxidase-stained section, the transported label can be seen to lie preferentially in blobs. In all eight of the blob injections, all of the surrounding blobs seemed to be labeled, with no subpopulation of blobs or subregion of each individual blob preferentially labeled.

Five of the injections lay very close to, but not in, blobs. For these, the transported label also lay near, but not in, blobs. In the eight injections that spanned blob and nonblob regions, the transported label was still patchy but lay in both blob and nonblob regions.

Even though the injections were centered in layers 2-3, most of the injections resulted in transported label throughout the thickness of the cortex. Both blob and nonblob injections had the same laminar pattern of labeling, except that one of the nonblob injections resulted in a few small labeled cells in layer $4 \mathrm{C} \beta$. Figure 3 shows the sections through the entire thickness of the cortex for the injection shown in Figure 1d, a blob injection. For both blob and nonblob injections, patchy label in layers $2-3$ corresponded to the core of the injection, predominantly blob or nonblob. The label in layer $4 \mathrm{C}$ was not patchy; it was confined to a small circular region lying directly under the core of the injection site and was made up of tiny clumps of grains not in cell bodies, but apparently in vertically running processes which we presume to be the descending axons of the layer 2-3 cells in the injection site. In layer 5, labeled axons (possibly collaterals of those running verticaly through layer 4C) fanned out again and ended in patches of diffuse label that were in vertical alignment with the patches in layers $2-3$. These patches, while clear on the original slides, were too faint to be easily or convincingly reproduced, and they do not show up well in Figure 3. Several large labeled cell bodies were usually seen in the center of the labeled region (directly below the injection site) in layer 5 .

\section{Discussion}

Within area 17 , blobs evidently connect preferentially to blobs, and nonblobs connect to nonblobs. We previously found that blob and nonblob regions of area 17 project to different regions of area 18, suggesting the existence of two anatomically segregated pathways. The results presented here suggest that these two pathways are not only segregated in their connections to area 18 , but that even in area 17 they do not influence each other strongly.

These results may seem surprising in view of two previous reports, that of Rockland and Lund (1983) and our own (Livingstone and Hubel, 1984), both of which studied the intrinsic connections of area 17 using horseradish peroxidase axonal transport labeling (one, wheat germ-conjugated horseradish peroxidase, the other ordinary horseradish peroxidase), and both of which gave results different from the present ones and different from each other. Around their injections, Rockland and Lund (1983) obtained a lattice of label that included the blobs in its walls, whereas our label was primarily restricted to the nearby blobs. The main difference between these results and our present ones is in the size of the injections. Whereas the earlier studies involved injections almost an order of magnitude larger and, consequently, clearly including both blobs and nonblob regions and sometimes occupying the full thickness of the cortex, in the present work our injections were confined either to blobs or to interblob regions and limited to layers 2-3. From our present results, much larger injections might be expected to give labeling both of the surrounding blobs and the matrix of nonblob tissue. Our first thought would be not to expect patchy labeling of nonblob cortex, since the injection sites would surely include cells of all orientation affiliations (however, see Mitchison and Crick, 1982). Thus one's first expectation would be that a large injection would produce uniform labeling of surrounding tissue. The fact that neither study gave such a result is something we cannot explain, let alone the divergent results that were obtained. But given the profound differences between blob cells and nonblob cellsin physiology, in their thalamic inputs and their connections to area 18, and in staining for cytochrome oxidase and labeling with deoxyglucose (Humphrey and Hendrickson, 1983)-one should perhaps not be too surprised to find differences in their abilities to take up and transport various substances. That the manifestations of these differences differed in the two earlier studies is most likely due to technical factors: ordinary versus wheat germ-coupled horseradish peroxidase, the vagaries of 
(2)

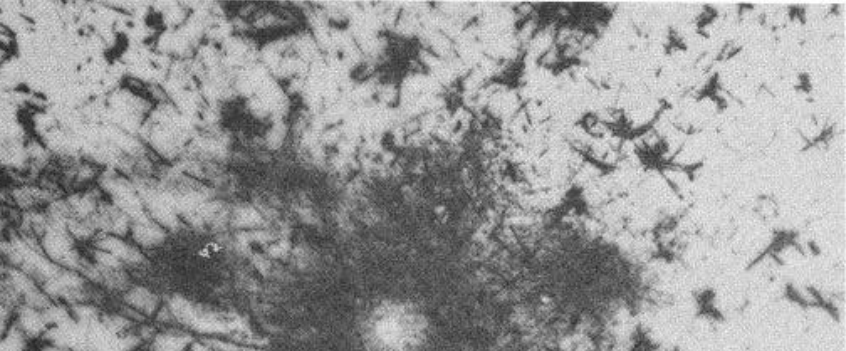

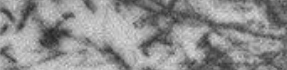

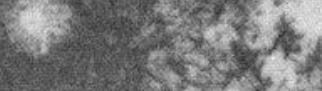
$\cos x^{2}$ is $x^{-}$

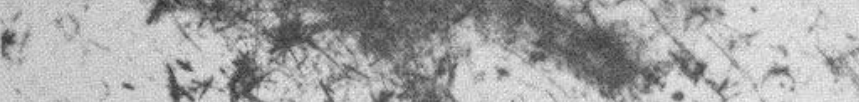

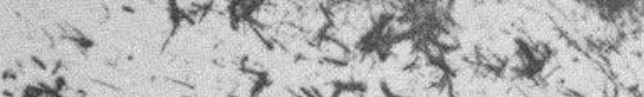

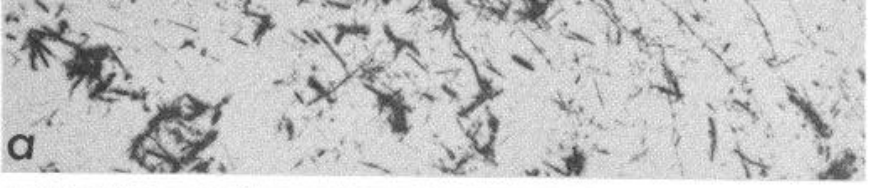

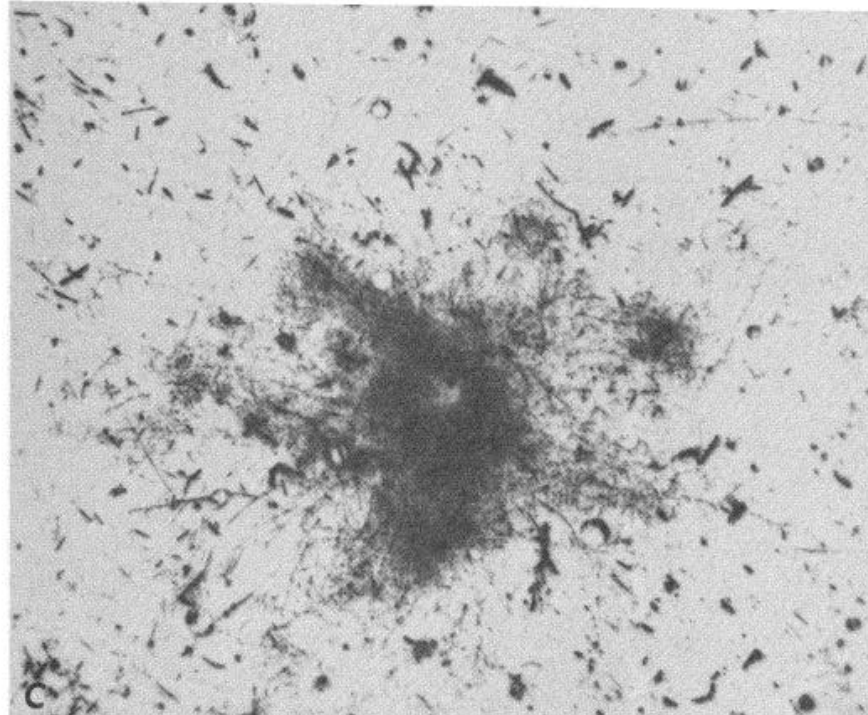

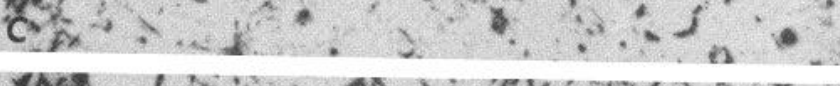

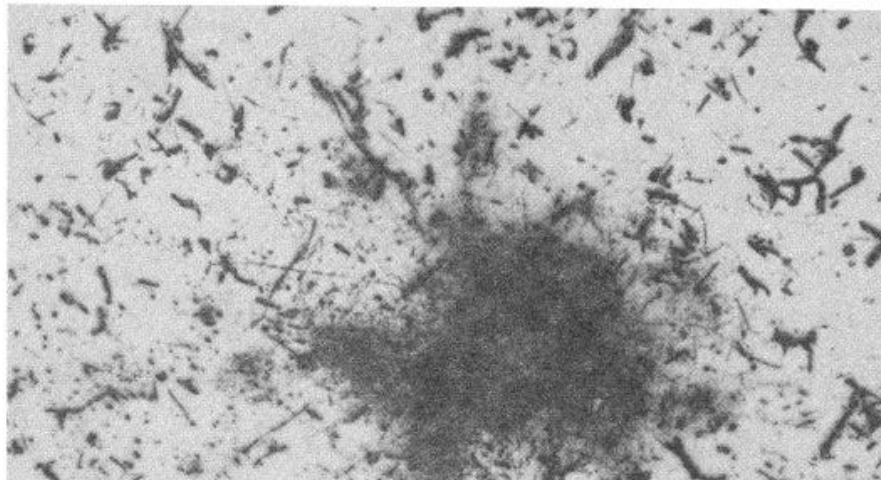

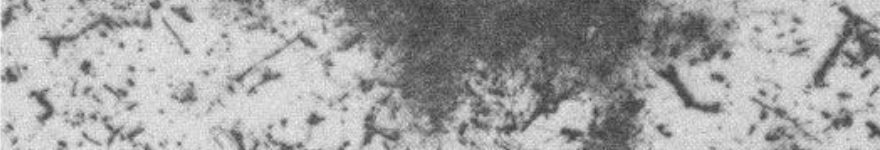

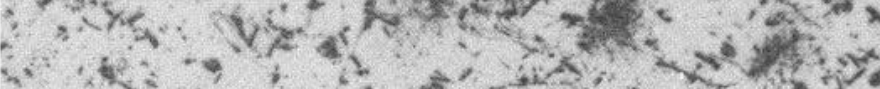

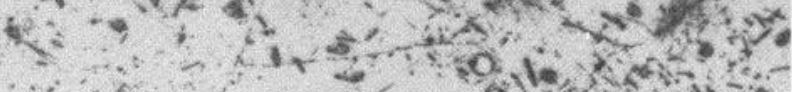

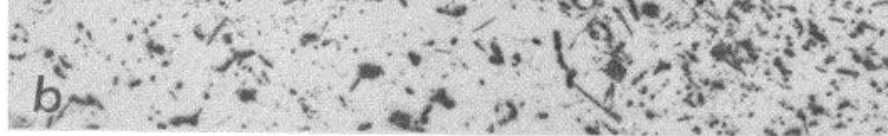

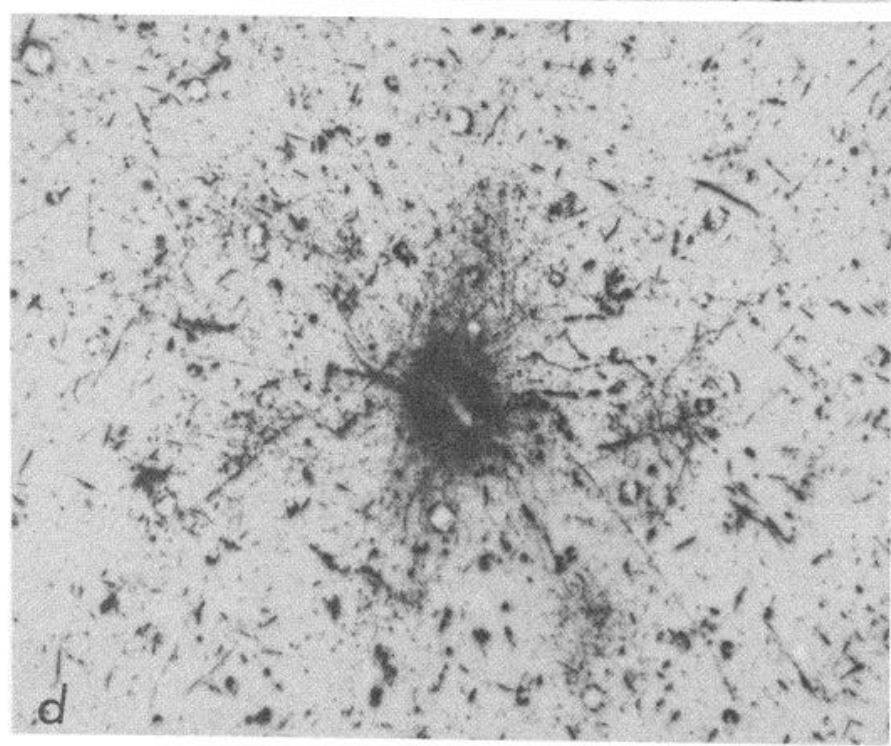

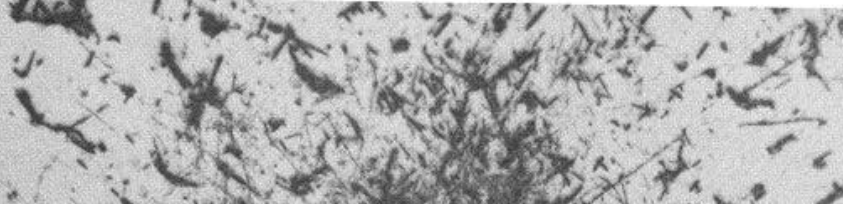

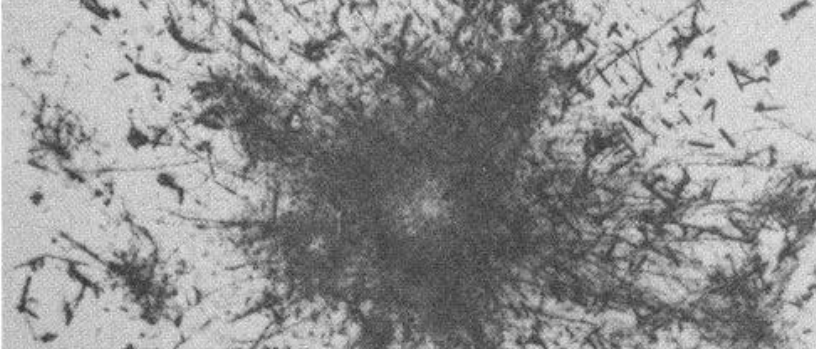

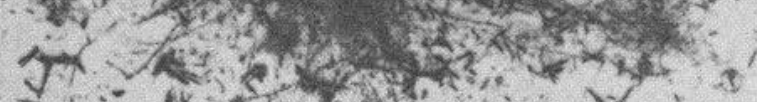

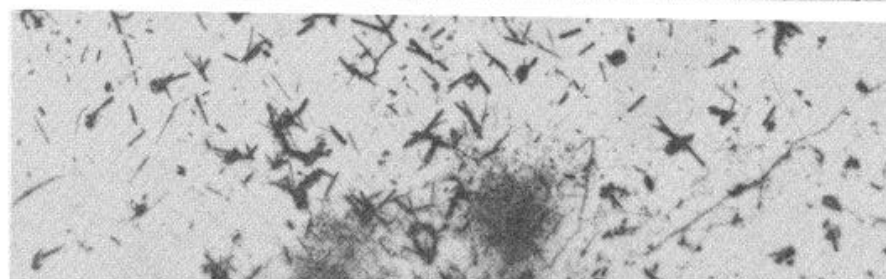

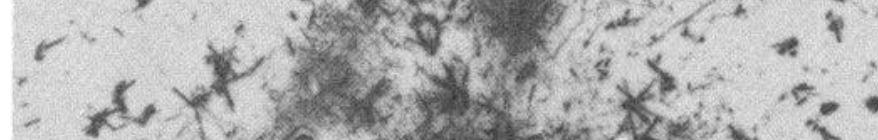

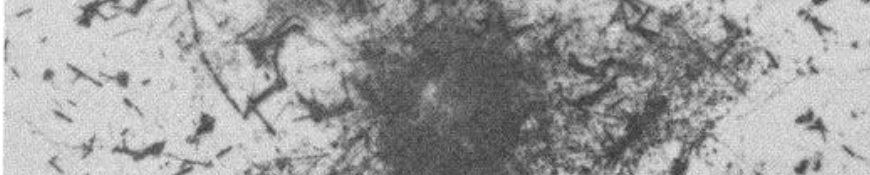

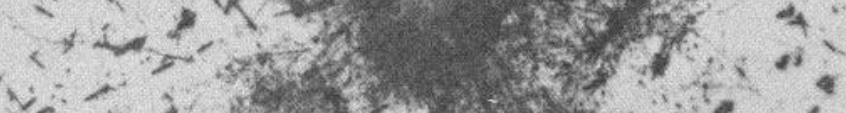

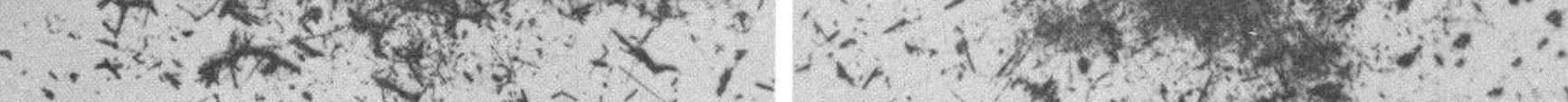

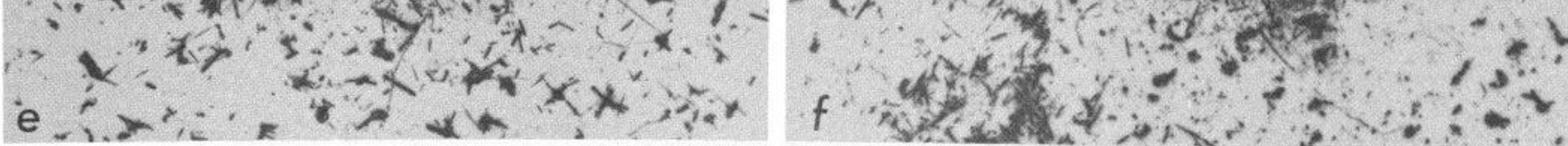




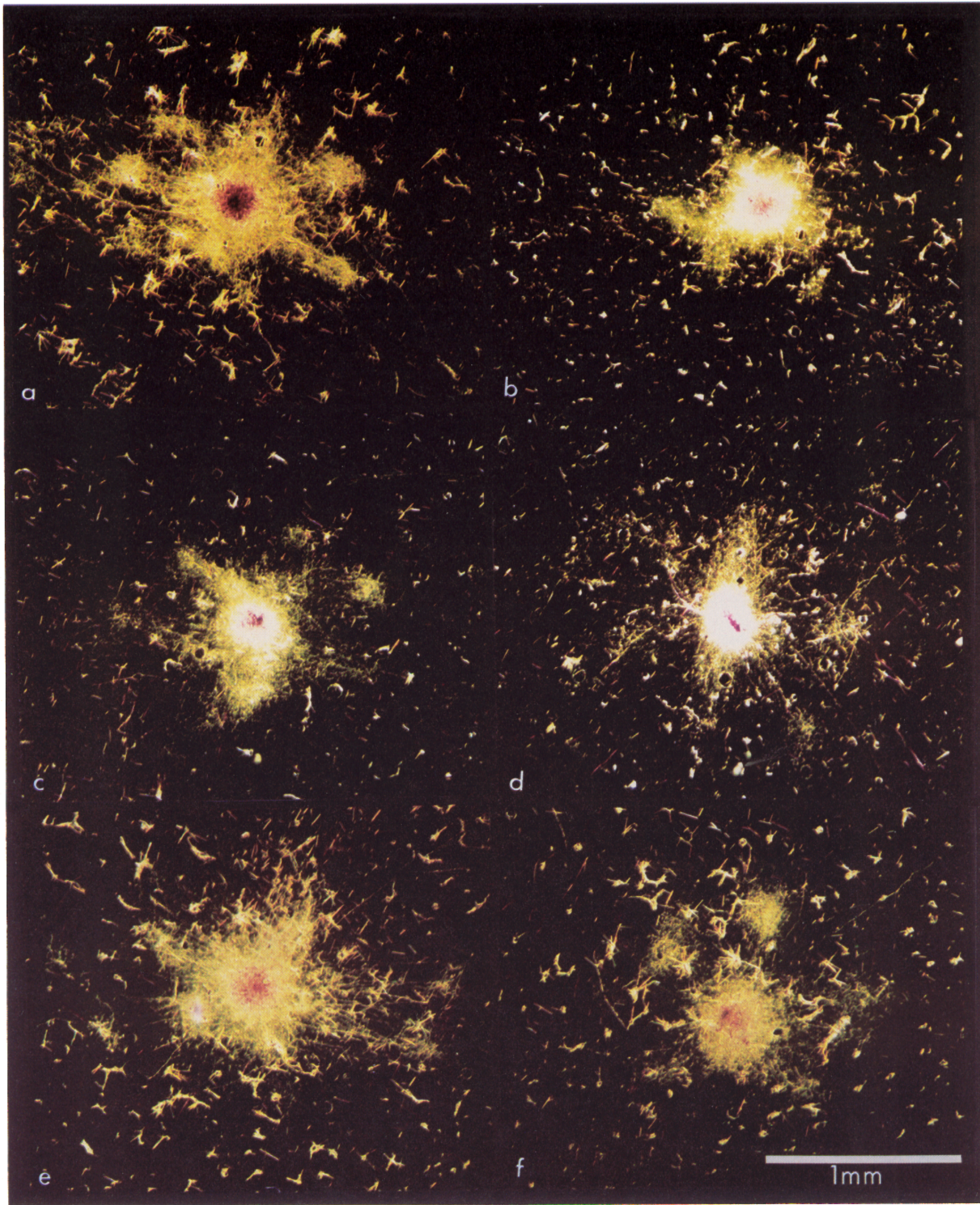

Figure 1. Six horseradish peroxidase injections in layers 2-3 of macaque area 17 . The sections were reacted with tetramethylbenzidine (Mesulam, 1982) and photographed through crossed polarizers. The acetate overlay is a negative image of the same sections. 

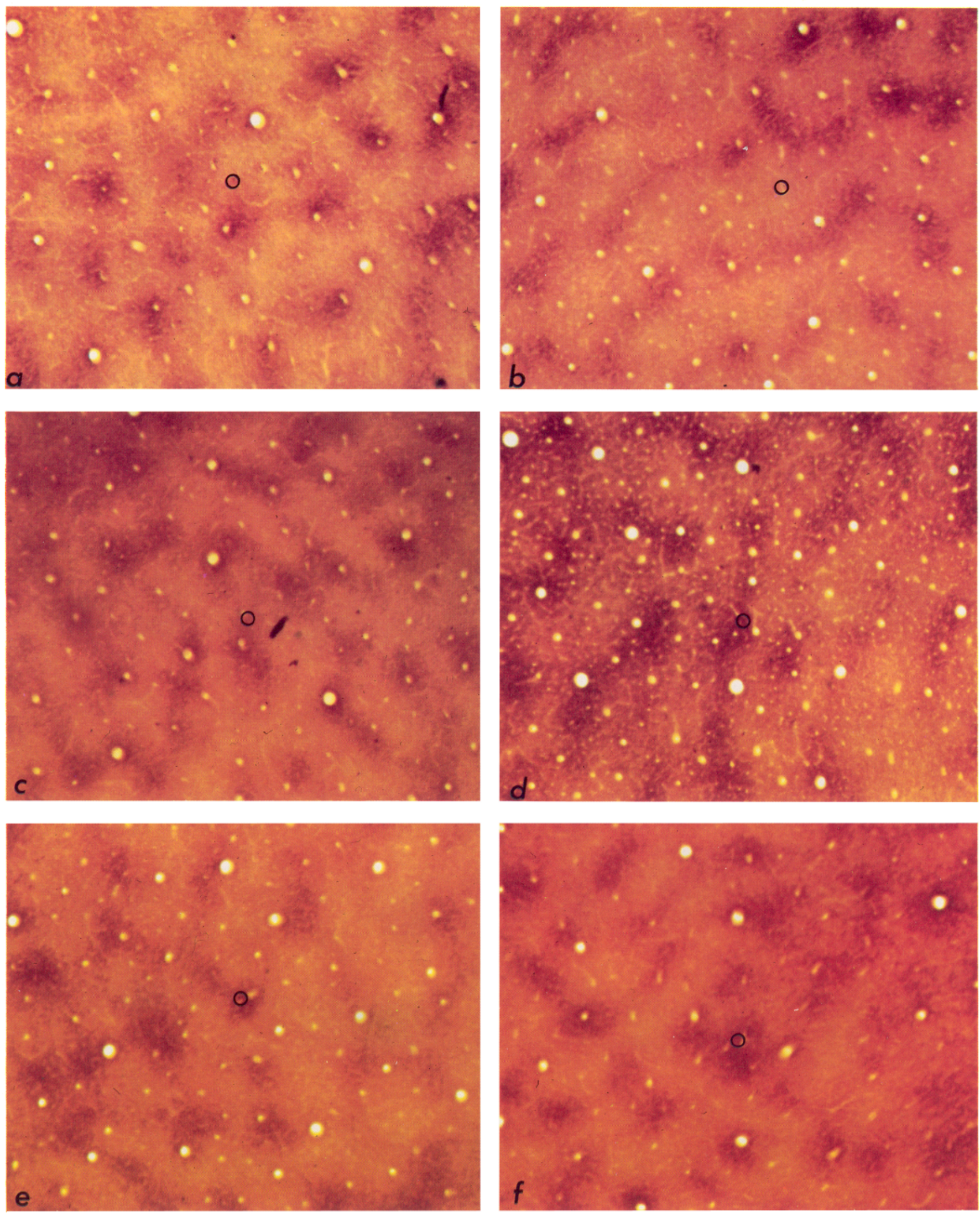

\section{$1 \mathrm{~mm}$}

Figure 2. Adjacent sections corresponding to the six injections in Figure 1, reacted for cytochrome oxidase (Wong-Riley, 1979). The magnification and orientation are identical to those of Figure 1. The acetate overlay should be aligned so that the letters $a$ to $f$ correspond precisely. (If there is no acetate overlay, the reader is encouraged to trace the injections in Figure 1 on a piece of clear plastic and compare it to Fig. 2.) 


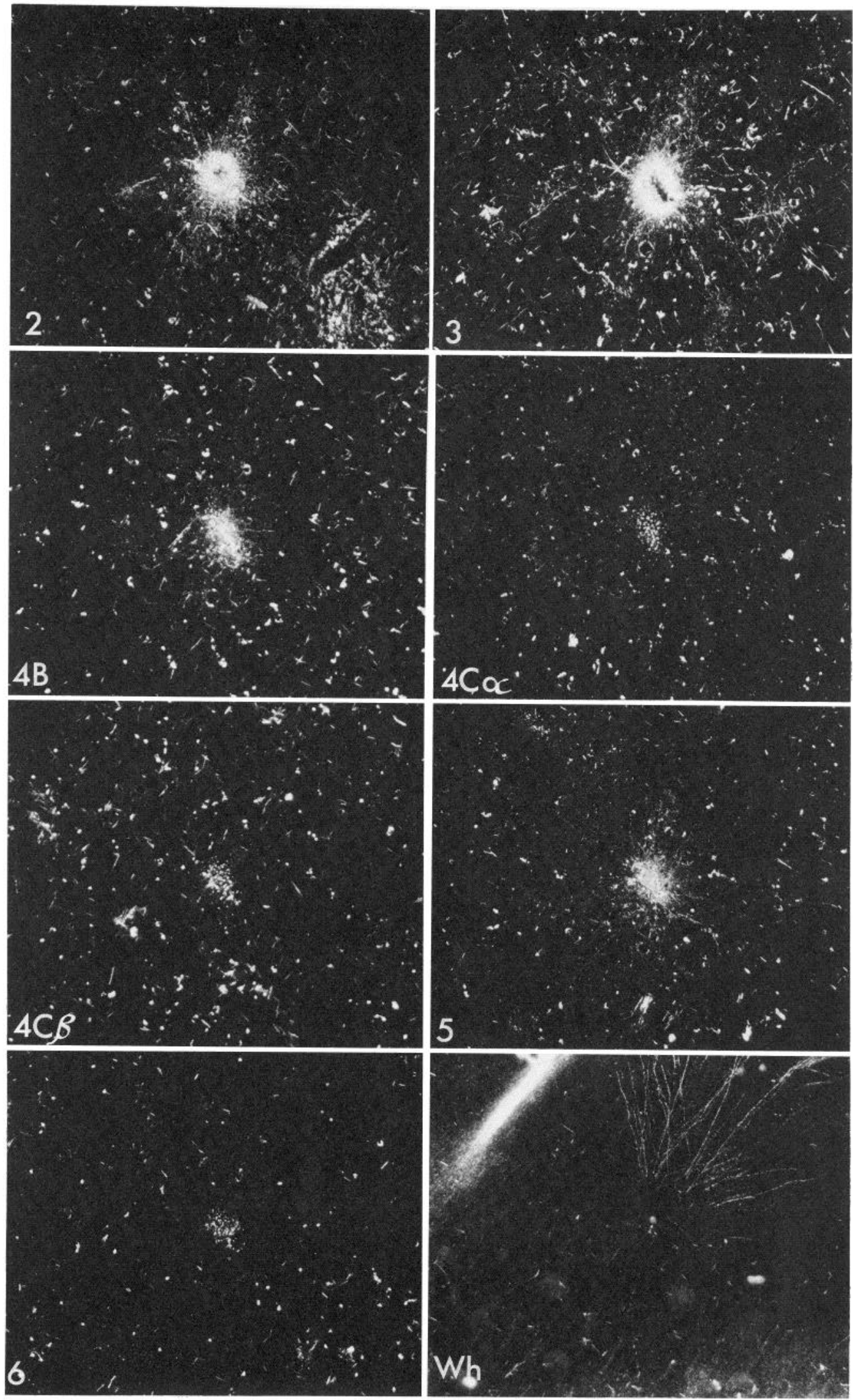

Figure 3. Sections through the full thickness of cortex of the same injection shown in Figure 1d. The sections were reacted with tetramethylbenzidine and photographed through crossed polarizers. The layer of each section is indicated by numbers and letters on the figure. Each section is in exactly the same orientation. The fibers in the white matter $(W h)$ are heading anteriorly, toward area 18. 
perfusion methods, times of survival, depths of injections, the characteristics of the injection medium, and myriad others.

It should be stressed that our conclusion that blob cells project preferentially to blobs and nonblob cells to nonblob cortex applies only to regions a few hundred micrometers from the injection core. Concerning the halo of label immediately surrounding the core we can obviously say nothing, and thus we cannot exclude blob-to-nonblob connections within that distance.

Our physiological recordings from area 17, although showing marked differences in response properties of blob and nonblob cells in layers 2 and 3 , are not in themselves enough to indicate a total mutual insulation of the two systems. The lack of orientation of blob cells would be compatible with nonblob-toblob projections if each blob cell were to receive convergent input from nonblob cells representing all orientation affiliations. But any such convergence should lead to a selective responsiveness to lines without any selectiveness to orientation, instead of the straightforward center-surround or center-only properties actually observed in blob cells. Thus the lack of an anatomically demonstrable nonblob-to-blob projection can be readily rationalized. There are some hints from the physiology that the reverse connections, from blob cells to nonblob cells, might exist, since a few of the nonblob cells that show orientation selectivity nevertheless respond best to slits of restricted wavelength composition, preferring, for example red to white. Such properties could be best understood as the result of convergent input from double-opponent blob cells.

The possible function of the lateral connections we do see can still only be speculated on. Patchiness of the nonblob projections can be interpreted in terms of connectedness of cells of like orientation-excitatory, for building up complex receptive fields, inhibitory for end stopping-or in terms of inhibitory connectedness of cells with different orientations, designed to sharpen orientation tuning. Blob-to-blob connections might be involved in building up double opponent fields from center-only (type 2) fields by supplying the necessary opponent surround inhibition, and again such connections could be either excitatory or inhibitory. Or perhaps (as we have discussed before) the double opponent properties are concocted in the geniculate (or the retina), and the cortical connections are there to potentiate the receptive field surrounds, or to make adjustments so that diffuse light and white light are more nearly ineffective. We saw no hint of connections preferentially to alternate ocular dominance columns, as might be expected from the fact that blobs are centered along ocular dominance columns and contain cells with strong eye preferencc.

If blobs are connected to nearby blobs, serially across the entire cortex (as horizontal cells and amacrine cells are connected across the retina), one might expect the receptive fields to be large, perhaps with small centers and very extensive surrounds. That would be welcome in view of the wide spatialcolor interactions known to exist psychophysically. On the contrary, the cells we have tested for this (eccentricity 0 to $6^{\circ}$ ) seem to have fields whose surrounds are confined to a few degrees of visual angle, a distance whose magnitude is the same order as that corresponding to the projections we observe: a few millimeters. We therefore suspect that the wide interactions seen psychophysically depend on connections existing beyond area 17.

\section{References}

Gilbert, C. D., and T. N. Wiesel (1979) Morphology and intracortical projections of functionally identified neurons in cat visual cortex. Nature 280: $120-125$.

Gilbert, C. D., and T. N. Wiesel (1983) Clustered intrinsic connections in cat visual cortex. J. Neurosci. 3: 1116-1133.

Hendrickson, A. E., S. P. Hunt, and J. -Y. Wu (1981) Immunocytochemical localization of glutamic acid decarboxylase in monkey striate cortex. Nature 292: 605-607.

Horton, J. C., and D. H. Hubel (1981) A regular patchy distribution of cytochrome-oxidase staining in primary visual cortex of the macaque monkey. Nature 292: 762-764.

Hubel, D. H., and T. N. Wiesel (1962) Receptive fields, binocular interaction and functional architecture in the cat's visual cortex. $\mathbf{J}$. Physiol. (Lond.) 160: 106-154.

Humphrey, A. L., and A. E. Hendrickson (1983) Background and stimulus-induced patterns of high metabolic activity in the visual cortex (area 17) of the squirrel and macaque monkey. J. Neurosci. 3: $345-358$.

Livingstone, M. S., and D. H. Hubel (1982) Thalamic inputs to cytochrome oxidase-rich regions in monkey visual cortex. Proc. Natl. Acad. Sci. U. S. A. 79: 6098-6101.

Livingstone, M. S., and D. H. Hubel (1984) Anatomy and physiology of a color system in the primate visual cortex. J. Neurosci. 4: $309-$ 356.

Lorente de Nó, R. (1933) Studies on the structure of the cerebral cortex. J. Psychol. Neurol. 45: 382-438.

Mesulam, M. -M. (1982) Principles of horseradish peroxidase neurohistochemistry and their applications for tracing neural pathwaysAxonal transport, enzyme histochemistry and light microscopic analysis. In Tracing Neural Connections with Horseradish Peroxidase, M. -M. Mesulam, ed., pp. 1-151, John Wiley \& Sons, Inc., New York.

Mitchison, G., and F. Crick (1982) Long axons within the striate cortex: Their distribution, orientation, and patterns of connection. Proc. Natl. Acad. Sci. U. S. A. 79: 3661-3665.

Mountcastle, V. B. (1957) Modality and topographic properties of single neurons of cat's somatic sensory cortex. J. Neurophysiol. 20: 408434.

Rockland, K. S., and J. S. Lund (1983) Intrinsic laminar lattice connections in primate visual cortex. J. Comp. Neurol. 216: 303-318.

Wong-Riley, M. T. T. (1979) Changes in the visual system of monocularly sutured or enucleated cats demonstrable with cytochrome oxidase histochemistry. Brain Res. 171: 11-28. 